\title{
The efficacy and safety of a proprietary onion-pumpkin extract (OPtain120) on blood pressure: an open-label study
}

\author{
Jay Udani ${ }^{1}$, Orie Yoshinari ${ }^{2 *}$, Hiroyoshi Moriyama ${ }^{3}$, Yoshiaki Shiojima $^{2}$, Xiaoming Chien $^{4}$ \\ ${ }^{1}$ Northridge Hospital, 18300 Roscoe Blvd, Northridge, CA 91325, USA; ${ }^{2}$ Ryusendo Co., Ltd., \\ 1-5-3 Nishi-ikebukuro, Toshima-ku, Tokyo 171-0021, Japan; ${ }^{3}$ The Japanese Institute for Health \\ Food Standards, 6-26-12 Hongo, Bunkyo-ku, Tokyo 113-0033, Japan; ${ }^{4}$ The Horn Company, \\ 16050 Canary Avenue, La Mirada, CA 90638, USA
}

*Corresponding Author: Orie Yoshinari, PhD, Ryusendo Co., Ltd., 1-5-3 Nishi-ikebukuro, Toshima-ku, Tokyo 171-0021, Japan

Submission Date: May 20, 2015, Acceptance date: June 26, 2015: Publication date: June 28, 2015

\begin{abstract}
Background: Nutraceuticals and functional foods are increasingly being used to help manage hypertension. Treatment with either pumpkin or onion can significantly lower systolic and diastolic blood pressure in animal studies. Traditionally, pumpkin has been used to support healthy blood pressure, glucose tolerance and lipid levels. Onion contains high levels of flavonoids, including quercetin, which decreases blood pressure and promotes restoration of healthy endothelial function. However, human trials on these food sources are limited, and the combined effects of pumpkin and onion have not been examined yet.
\end{abstract}

Objective: We performed an open-label clinical study to evaluate the effects of a proprietary onion-pumpkin extract (OPtain120) on systolic and diastolic blood pressure.

Methods: Healthy adults with systolic blood pressure (SBP) and diastolic blood pressure (DBP) in the elevated range of 140-159 and $80-90 \mathrm{mmHg}$, respectively, were enrolled in this study. Subjects consumed one capsule of onion-pumpkin extract twice daily for 12 weeks. Daily Home Blood Pressure Measurement (HBPM) was taken upon waking and before bed. Office Blood Pressure Measurement (OBPM) was taken in-clinic at Week 0, 6, and 12.

Results: 52 subjects were screened and 12 were enrolled in the study, with a total of 10 subjects completing the study. Systolic HBPM taken before bed demonstrated a statistically significant reduction from baseline $(147.23 \mathrm{mmHg})$ to Week $12(138.14 \mathrm{mmHg})$, representing a reduction of $9.09 \mathrm{mmHg}(6.17 \%, \mathrm{p}=0.021)$. Diastolic HBPM taken before bed demonstrated a decrease of $4.06 \mathrm{mmHg}(4.46 \%, \mathrm{p}=0.085)$, a significant reduction from baseline $(91.07 \mathrm{mmHg})$ at Week 12 
$(87.02 \mathrm{mmHg})$. Non-statistically significant reductions were seen in the early morning Systolic $(3.14 \%)$ and Diastolic (2.57\%) HBPM and in the Systolic (1.36\%) OBPM.

Conclusion: OPtain120 was safely consumed over a 12-week period. OPtain 120 appears to be effective in lowering Systolic Blood Pressure at bedtime in healthy individuals with slightly elevated blood pressure. This study suggests that onion-pumpkin extract may aid individuals who manage their cardiovascular risk factors with diet and lifestyle.

Key Words: pumpkin, onion, extract, blood pressure

\section{INTRODUCTION}

Hypertension creates a serious public health challenge. It is not only highly associated with morbidity and mortality, but has also become one of the most common diseases in the world [1]. In the United States alone, one fourth of adults have high blood pressure, with an even higher incidence for at-risk populations, such as African-Americans and the elderly. Hypertension is the most important risk factor for cardiovascular diseases, including coronary heart disease, stroke, congestive heart failure, and peripheral vascular disease. Clinically, hypertension is characterized by abnormally high blood pressure with the systolic reading over $140 \mathrm{mmHg}$ and the diastolic reading greater than $90 \mathrm{mmHg}$ [1].

Lowering blood pressure has been demonstrated to cause $35 \%-40 \%$ reductions in the incidence of stroke; $20-25 \%$ in myocardial infarction, and up to 50\% in heart failure [1], thereby making hypertension a strong target for therapeutic intervention. Hypertension is managed primarily in two ways: lifestyle changes and antihypertensive medications. Lifestyle changes are typically the first line of treatment for hypertension, as they have been proven to be effective without side-effects. For example, weight reduction, increased physical activity and moderation of alcohol consumption have been shown to lower blood pressure. Dietary changes, including lowering sodium and incorporating fruits, vegetables, low-fat dairy products, whole grain, poultry fish and nuts with limited fats, red meat and sweets, are also recommended [1].

In addition to lifestyle changes, several classes of medications are available for treatment of severe hypertension. The antihypertensive drugs currently used in the clinic include angiotensin converting enzyme (ACE) inhibitors, angiotensin receptor blockers (ARBs), $\beta$-blockers (BBs), calcium channel blockers $(\mathrm{CCBs})$ and thiazide class diuretics. Among these treatments, the diuretic- based therapy was the most effective in preventing cardiovascular diseases, as demonstrated by the Antihypertensive and Lipid-lowering Treatment to prevent Heart Attack (ALLHAT) trial [1, 2]. However, these treatments are not typically recommended for mild hypertension, as medications expose the patient to increased health complications, due to side effects that outweigh clinical benefits. Consequently, nutraceuticals and food products have been increasingly investigated for their effects on hypertension. For example, omega-3 polyunsaturated fatty acids, omega-9 fatty acids, garlic, seaweed, and various vitamins and minerals have been shown to significantly reduce blood pressure [3].

Because dietary changes are a first-line intervention for hypertension, food products themselves are highly sought for the specific effects they may have on blood pressure. For 
example, onions contain high levels of the compound quercetin, which is particularly effective at lowering blood pressure, in addition to other flavonoids and natural compounds whose effects are not as well understood. Animal studies have provided evidence that onion has great potential in the treatment of hypertension in vivo. Rats on a high-fat, high-sucrose diet supplemented with Welsh onion had significantly lower blood pressure than their control counterparts. Urine and plasma readings of nitric oxide (NO) metabolites were increased, while NADH/NADPH oxidase and angiotensin II were suppressed. These molecular results suggest that the mechanism responsible for onion-dependent hypertension may be related to NO modulation [4, 5]. Although human data is sparse, one formulation containing onion has been clinically tested and shows great promise. A randomized, placebo-controlled, double-blind trial of a formulation containing onion and olive oil demonstrated significant decrease in arterial blood pressure and reduction in plasma viscosity 5 hours after administration [6].

Another food that holds great promise for combating hypertension is pumpkin. Pumpkin has been used as a traditional medicine and is known for its antidiabetic, antitumor, antibacterial, anti-inflammatory, and antihypertensive properties [7]. Modulation of hypertension or hypertensive risk factors by pumpkin product supplementation has been demonstrated in preliminary animal and human studies. Upon pumpkin seed oil supplementation in both nonovariectomized and ovariectomized rats, reduced blood pressure and lipid profile improvements were observed [8]. A recent clinical trial investigated the effects of supplemented pumpkin seed oil on hypertension and cholesterol levels in postmenopausal women. The results of this trial revealed that 12 -week administration of $2 \mathrm{~g}$ /day pumpkin seed oil led to a significant increase in HDL cholesterol and decrease in diastolic blood pressure [9]. Pumpkin fruit contains moderate to high alpha-glucosidase and ACE inhibitory activities, as measured by enzyme assays [10]. Because alpha-glucosidase inhibition reduces the impact of glucose on blood sugar, and ACE inhibition is used clinically to treat hypertension, further clinical trials should explore the effects of pumpkin on hypertension and metabolic homeostasis.

An open-label pilot study was designed in order to evaluate the safety and efficacy of a proprietary onion-pumpkin extract (OPtain120) for promoting healthy blood pressure. This study investigated the effects of Onion-Pumpkin extract on blood pressure, glucose metabolism, lipid levels, and antioxidant levels.

\section{METHODS}

\section{Investigational Product}

The investigational product for this study was proprietary Onion-Pumpkin Extract, OPtain 120 (Ryusendo Co., Ltd. Tokyo, Japan) (The nutrition composition of OPtain120 is shown in Table 1). The study product was GMP certified and taken twice daily, once before breakfast and once before dinner. During the course of the study, subjects were not allowed to consume drugs which might affect blood pressure, including but not limited to diuretics, ACE-inhibitors, beta blockers, angiotensin antagonists, and alpha-blockers. Permitted medications included hormonal contraception, including oral, patch, or devices. 
Table 1: Nutrition composition of OPtain 120

\begin{tabular}{|l|l|}
\hline Moisture & $3.5 \mathrm{~g} / 100 \mathrm{~g}$ \\
\hline Protein & $3.9 \mathrm{~g} / 100 \mathrm{~g}$ \\
\hline Lipid & $1.8 \mathrm{~g} / 100 \mathrm{~g}$ \\
\hline Ash & $2.5 \mathrm{~g} / 100 \mathrm{~g}$ \\
\hline Carbohydrate & $87.4 \mathrm{~g} / 100 \mathrm{~g}$ \\
\hline Sodium & $17.6 \mathrm{mg} / 100 \mathrm{~g}$ \\
\hline Calorie & $383 \mathrm{kcal} / 100 \mathrm{~g}$ \\
\hline
\end{tabular}

\section{Subject Population}

The study aimed encompassed healthy subjects with age of $\geq 18$ and Body Mass Index between 25 and $35 \mathrm{~kg} / \mathrm{m}^{2}$. Subjects had a Systolic Blood Pressure reading in the range of $140-160 \mathrm{mmHg}$ and Diastolic Blood Pressure reading from $80-90 \mathrm{mmHg}$. Subjects were recruited from the general population through online recruiting, advertising, and available clinical trial databases. Subjects were phone-screened prior to scheduling a screening visit. Females with childbearing potential agreed to use appropriate birth control methods during the entire study period. Subjects currently taking any antihypertensive medications or prescription blood pressure, cholesterol and/or diabetes medication were excluded from the study. Subjects taking dietary supplements or over the counter medications were allowed to undergo a 2-week washout. The inclusion and exclusion criteria are listed in Table 2.

Table 2: Inclusion and exclusion criteria used screening subjects for the study

\begin{tabular}{|l|l|}
\hline & $\begin{array}{l}\text { - Healthy volunteers } \geq 18 \text { years of age } \\
\text { - BMI } \geq 25 \text { and } \leq 35 \mathrm{~kg} / \mathrm{m}^{2}\end{array}$ \\
SBP: $140-160 \mathrm{mmHg}$ & DBP: $80-90 \mathrm{mmHg}$ \\
INCLUSION & Judged by the investigator to be in general good health on the basis of \\
CRITERIA & medical history \\
- Females of child bearing potential must agree to use appropriate birth \\
control methods during the entire study period. \\
- Understands the study procedures and signs forms providing informed \\
consent to participate in the study and authorization for release of relevant protected \\
health information to the study investigator. \\
- Subjects currently taking any antihypertensive medications \\
EXCLUSION \\
medication. \\
C Subjects taking prescription blood pressure, cholesterol and/or diabetes \\
medication (2 week washout allowed). \\
- History or presence of cancer in the prior two years. \\
- Recent history of (within 12 months) or strong potential for alcohol or \\
substance abuse.
\end{tabular}




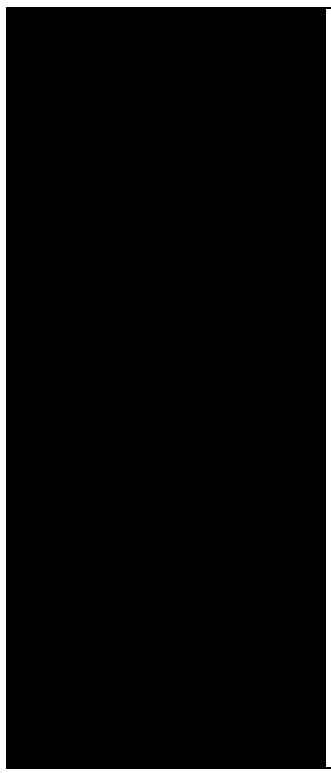

- Current tobacco smokers

- Participation in a clinical study with exposure to any non-registered drug product within 30 days prior.

- Individual has a condition the investigator believes would interfere with his or her ability to provide informed consent, comply with the study protocol, which might confound the interpretation of the study results or put the person at undue risk,

Including subjects who are Bed or wheelchair-bound.

- Clinically significant abnormal Physical Examination.

- Central Neurological disorders including but not limited to Spinal cord injuries, multiple sclerosis, and Parkinson disease.

- Pregnant, lactating, or unwilling to use adequate contraception during the duration of the study.

- Any other condition that, in the opinion of the investigator, would adversely affect the subject's ability to complete the study or its measures.

\section{Study Design}

This study was an open-label, pilot study (ClinicalTrials.gov Identifier: NCT02353104). This study was conducted at Northridge Hospital (Northridge, CA). The institutional review board (IRB) approval was received on July 26, 2013 by the MaGil IRB (Rockville, MD) prior to the initiation of study-related activities. Recruitment began on November 4, 2013 and ended on August 28, 2014. The trial concluded once the required number of subjects were enrolled and completed the study.

An in-clinic screening visit was conducted two weeks prior to the baseline visit. Subjects were instructed to fast before going to the clinic. At screening, subjects went through the informed consent process and were screened in the presence of all inclusion criteria and the absence of all the exclusion criteria. The screening process also included an interview on detailed medical history and medications, a physical examination, and an assessment of vital signs and anthropometric measurements, in addition to urine collection to run pregnancy tests for females with child bearing potential and blood collection for CBC and CMP tests. At the end of the visit, subjects were given a Blood Pressure device to be used two times every day, upon wakening and before bed, to record Home Blood Pressure Measurements (HBPM).

Subjects returned to the clinic at Week 0 (Baseline) after fasting for 10 hours, to conduct a review for intercurrent medical and concomitant medication history and adverse events reporting. Subjects also returned their blood pressure device, and those not consistently recording their measurements in their diary were reminded of their obligations regarding appropriate study compliance. Eligible subjects were assigned an enrollment number. Anthropometric measures and blood were drawn for the following laboratory tests: Fasting Glucose, Fasting Insulin, HbA1c, Lipid Panel, and 8-isoprostane, were also conducted. Office Blood Pressure Measurement (OBPM) was also taken in the clinic. At the end of the visit, subjects were dispensed with a 6-week supply of the study product, blood pressure devices for HBPM, and paper diaries, which served as a document of compliance and a record of 3-day food recall.

Subjects returned to the clinic at Week 6 and Week 12 for repeat assessments. The duration of the study took up to 14 weeks for each subject. 


\section{Endpoints}

The study's objectives were to evaluate the effect of the Onion-Pumpkin Extract in supporting healthy blood pressure, healthy glucose metabolism, healthy lipid levels, and healthy antioxidant levels. Endpoints included Changes in Home and Office Systolic Blood Pressure and Diastolic Blood Pressure measurements, Changes in Fasting Glucose, Fasting Insulin, and HbA1c, Changes in Total Cholesterol Low-density (LDL) Cholesterol, High-density (HDL) Cholesterol, and Change in 8-Isoprostane. The safety and tolerability of the study product was also assessed through Changes in CBC, CMP, and adverse event analysis.

\section{Statistics}

A parallel dual data entry was completed by data management personnel across all endpoints. Data validation and reconciliation of parallel entry occurred after the dual data entry process. The monitoring team compared the values on the original CRFs or source documents, correcting any discrepancies found. All data elements were screened for reasonableness, and every missing, suspicious, or impossible value was referred back to the monitoring team for query generation and resolution. The database was formally locked after all suspicious entries in the database were resolved.

All variables under investigation were summarized by time point. Endpoints in interval/ratio scale were presented ( $\mathrm{n}$, mean, standard deviation and standard error). Numerical variables were presented graphically, as plots of average value versus time. The Paired Sample t-test was used to assess within-group changes from Baseline to each subsequent time point to variables which were normally distributed. For non-normally distributed data, the Wilcoxon Signed Ranks test or Sign test were used to assess within-group changes from Baseline to each subsequent time point. Percentage Changes in each succeeding time point were used to quantify increase or decrease of endpoints versus Baseline.

Safety and tolerability of Onion-Pumpkin Extract based on CBC and CMP read from Baseline to succeeding time points were assessed by Paired Sample t-test for data identified as normally distributed or by the non-parametric Wilcoxon Signed Ranks test or Sign test for data that was substantially non-normally distributed.

All tests of hypotheses were done at alpha=0.05. All analysis were done using SPSS version 19.

A Modified per Protocol (mPP) analysis was performed to assess the efficacy variables of the study. The mPP population, consisting of all subjects randomly assigned to one of the treatments, was analyzed together, regardless of whether or not they completed the whole study. This analysis also ignores non-compliance, protocol deviations, withdrawal, and anything that happens after enrollment.

\section{RESULTS}

52 subjects were screened, and 12 enrolled in the study. Two did not complete follow up and a total of 10 subjects completed the study (Figure 1). The demographics and baseline characteristics are shown in Table 3. 


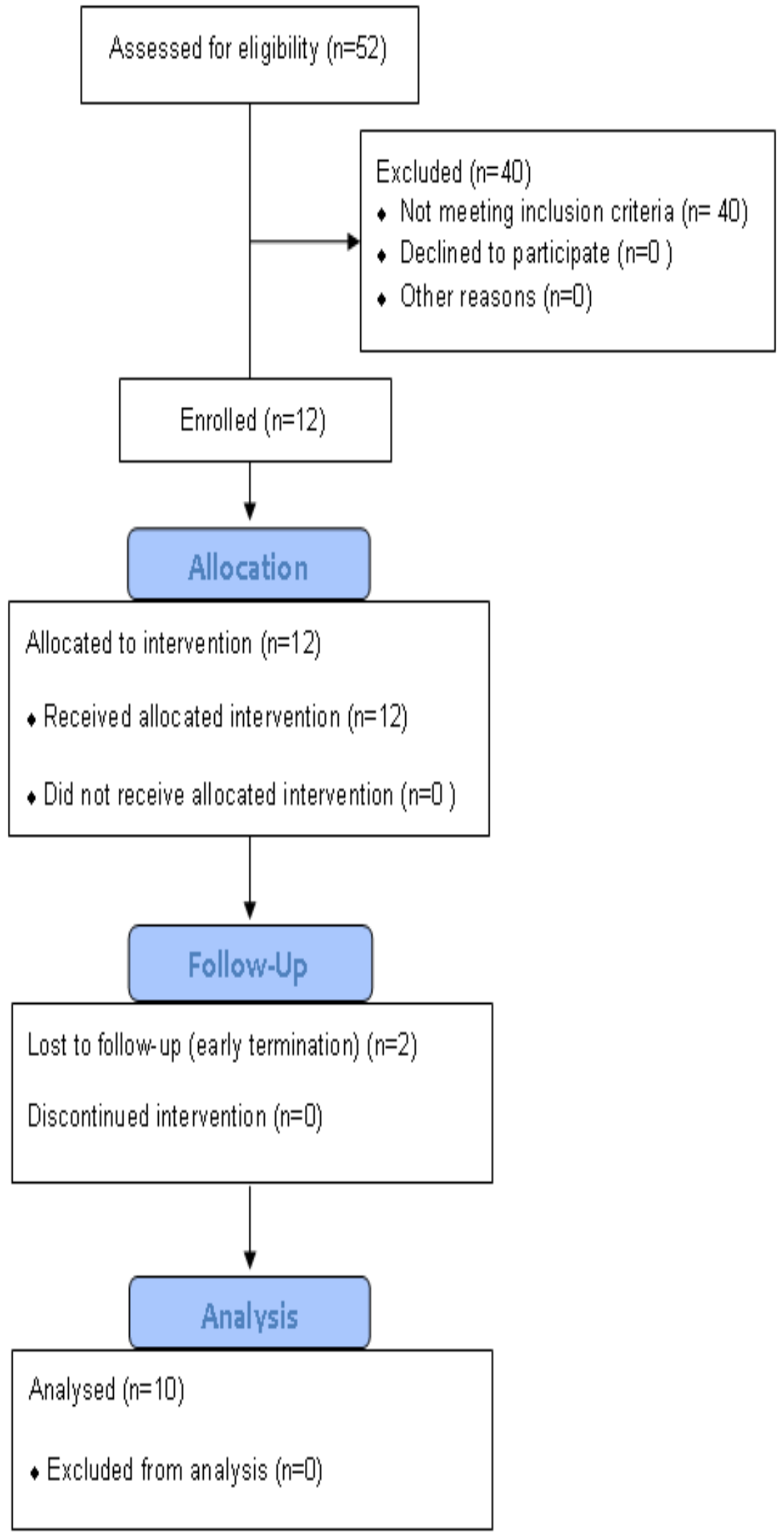

Figure 1. Attrition Chart 
Table 3: Demographic and baseline characteristics

\section{Baseline Characteristics}

\begin{tabular}{|c|c|}
\hline $\mathrm{n}$ (Total number of enrolled, inclusive of early terminated) & 12 \\
\hline Male & 8 \\
\hline Female & 2 \\
\hline Age - Mean & 50.40 \\
\hline Age - Range & $50.40 \pm 8.07$ \\
\hline Weight - Mean (kg) & 91.23 \\
\hline Weight - Range (kg) & $91.23 \pm 15.57$ \\
\hline Height - Mean (cm) & 171.63 \\
\hline Height - Range (cm) & $171.63 \pm 10.92$ \\
\hline BMI - Mean $\left(\mathrm{kg} / \mathrm{m}^{2}\right)$ & 30.82 \\
\hline BMI - Range $\left(\mathrm{kg} / \mathrm{m}^{2}\right)$ & $30.82 \pm 3.83$ \\
\hline \multicolumn{2}{|l|}{ Marital Status } \\
\hline Single & 3 \\
\hline Married & 5 \\
\hline Divorced & 1 \\
\hline Separated & 1 \\
\hline \multicolumn{2}{|l|}{ Ethnicity } \\
\hline Latino/Hispanic & 2 \\
\hline Asian & 2 \\
\hline Caucasian & 6 \\
\hline
\end{tabular}

The results provided were for the 10 subjects. The primary objective was to evaluate the effect of the study product on blood pressure. Systolic HBPM taken before bed demonstrated a statistically significant reduction from baseline $(147.23 \mathrm{mmHg})$ to Week $12(138.14 \mathrm{mmHg})$, corresponding to a reduction of $9.09 \mathrm{mmHg}(6.17 \%$; $\mathrm{p}=0.021)$ (Figure 2). Moreover, Diastolic HBPM taken before bed demonstrated a nearly significant reduction of $4.06 \mathrm{mmHg}(4.46 \%$; $\mathrm{p}=0.085)$ from baseline $(97.07 \mathrm{mmHg})$ to Week $12(87.02 \mathrm{mmHg}$ ) (Figure 3) (Table 4). 


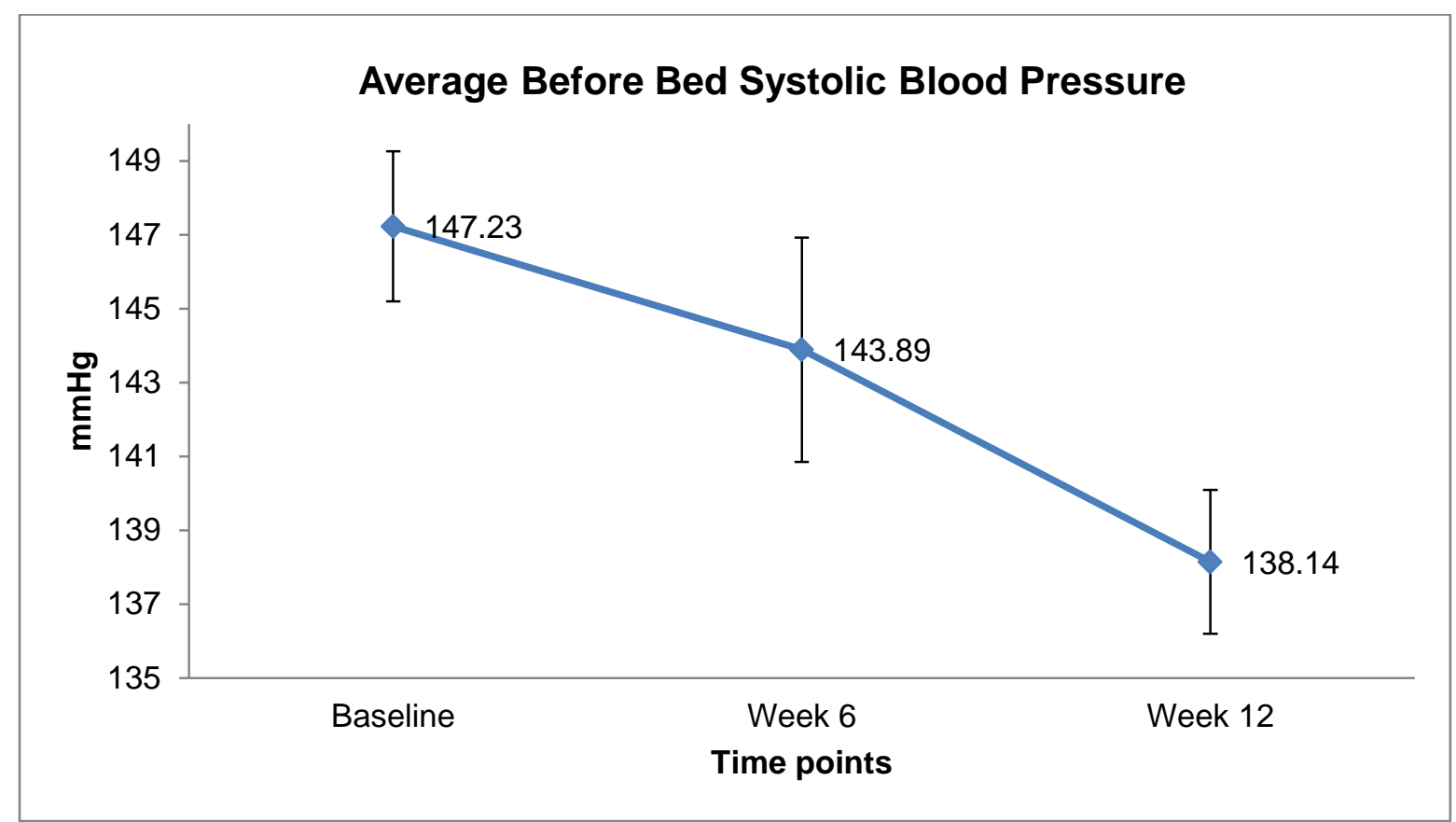

Figure 2. Comparison between baseline and Week 6 and 12 in Home Blood Pressure Measurements of Before Bed Systolic Blood Pressure.

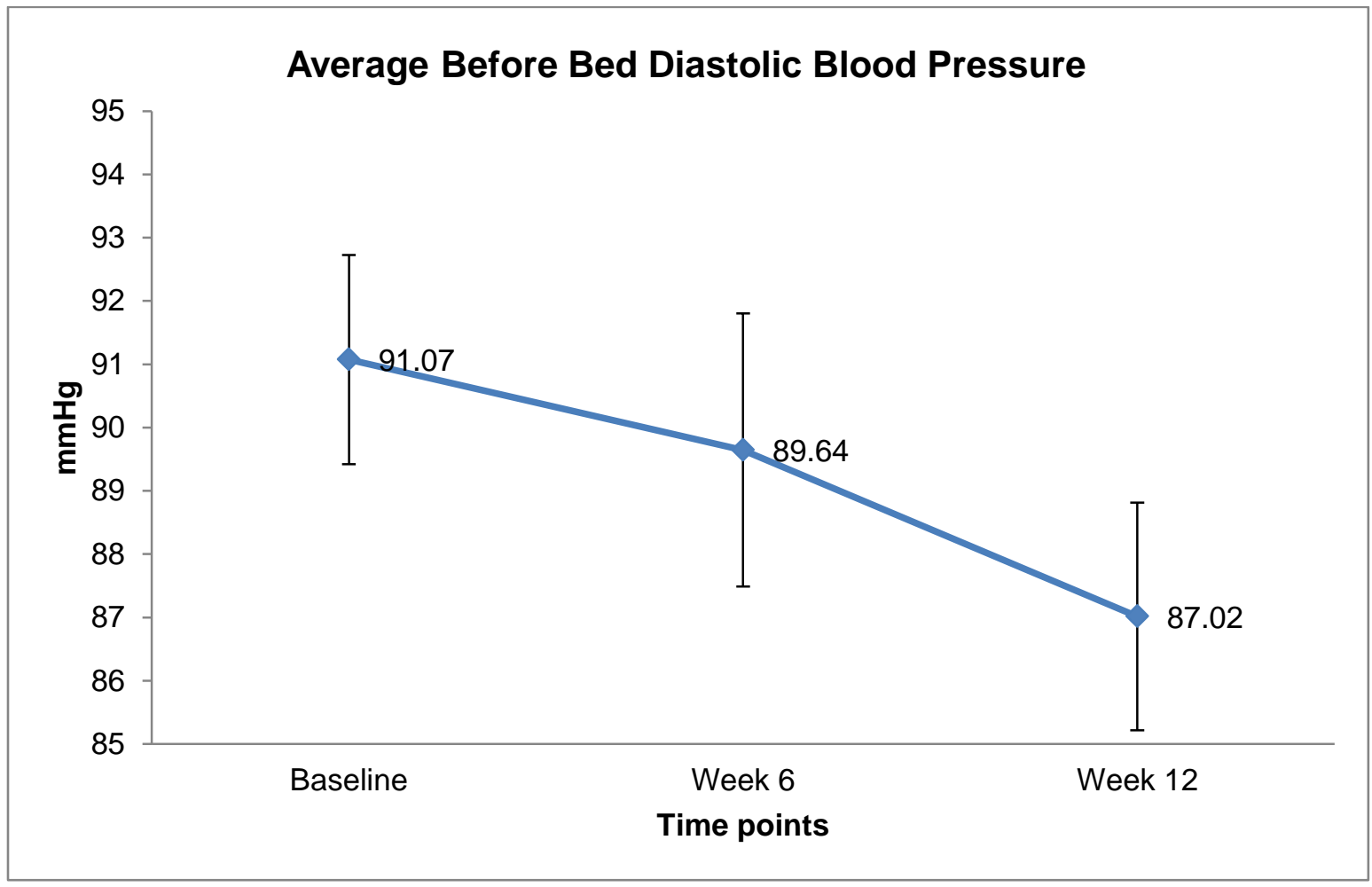

Figure 3. Comparison between baseline and Weeks 6 and 12 in Home Blood Pressure Measurements of Before Bed Diastolic Blood Pressure. 
Table 4: Comparison between baseline and Week 12 in Home Blood Pressure Measurements (HBPM)

\begin{tabular}{|c|c|c|c|c|}
\hline $\begin{array}{l}\text { HOME BLOOD PRESSURE } \\
\text { MEASUREMENT (HBPM) }\end{array}$ & Mean & $\begin{array}{c}\text { Std. } \\
\text { Deviation }\end{array}$ & Sig. & $\begin{array}{l}\text { Percent } \\
\text { Change } \\
\text { (versus } \\
\text { Baseline) }\end{array}$ \\
\hline \multirow{2}{*}{ Before Bed Systolic HBPM } & 147.23 & 6.430 & \multirow{2}{*}{$0.021 *$} & \multirow{2}{*}{$-6.17 \%$} \\
\hline & 138.14 & 6.159 & & \\
\hline \multirow{2}{*}{ Before Bed Diastolic HBPM } & 91.07 & 5.223 & \multirow{2}{*}{0.085} & \multirow{2}{*}{$-4.46 \%$} \\
\hline & 87.02 & 5.693 & & \\
\hline \multirow{2}{*}{ Wake-Up Systolic HBPM } & 143.73 & 13.062 & \multirow{2}{*}{0.109} & \multirow{2}{*}{$-3.14 \%$} \\
\hline & 139.21 & 5.776 & & \\
\hline \multirow{2}{*}{ Wake-up Diastolic HBPM } & 91.07 & 9.647 & \multirow{2}{*}{0.549} & \multirow{2}{*}{$-2.57 \%$} \\
\hline & 88.72 & 6.231 & & \\
\hline
\end{tabular}

There were also non-statistically significant reductions in early morning Systolic (3.14\%) and Diastolic (2.57\%) (Figure 4 and 5) HBPM and in the Systolic (1.36\%) OBPM (Figure 6) (Table 5).

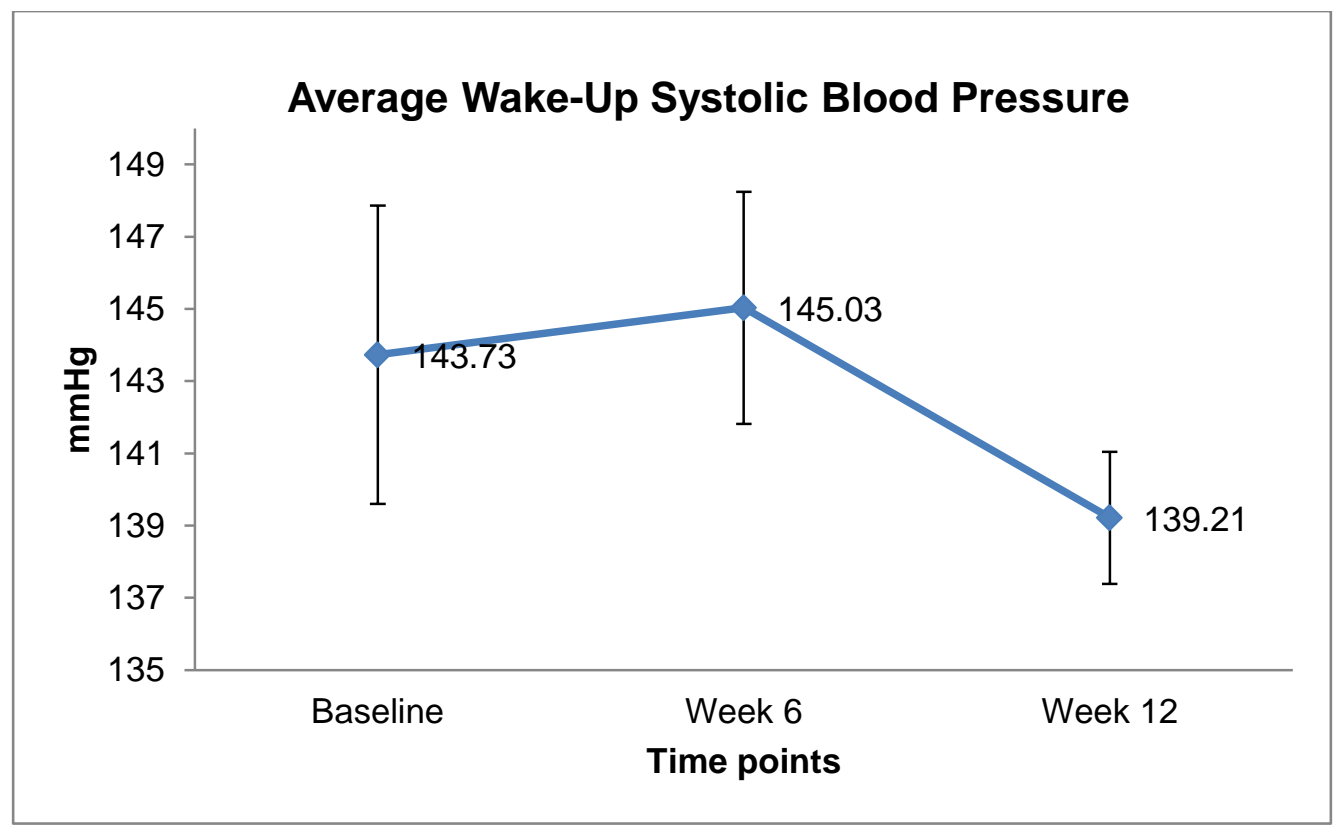

Figure 4. Comparison between baseline and Week 6s and 12 in Home Blood Pressure Measurements of Wake-Up Systolic Blood Pressure 


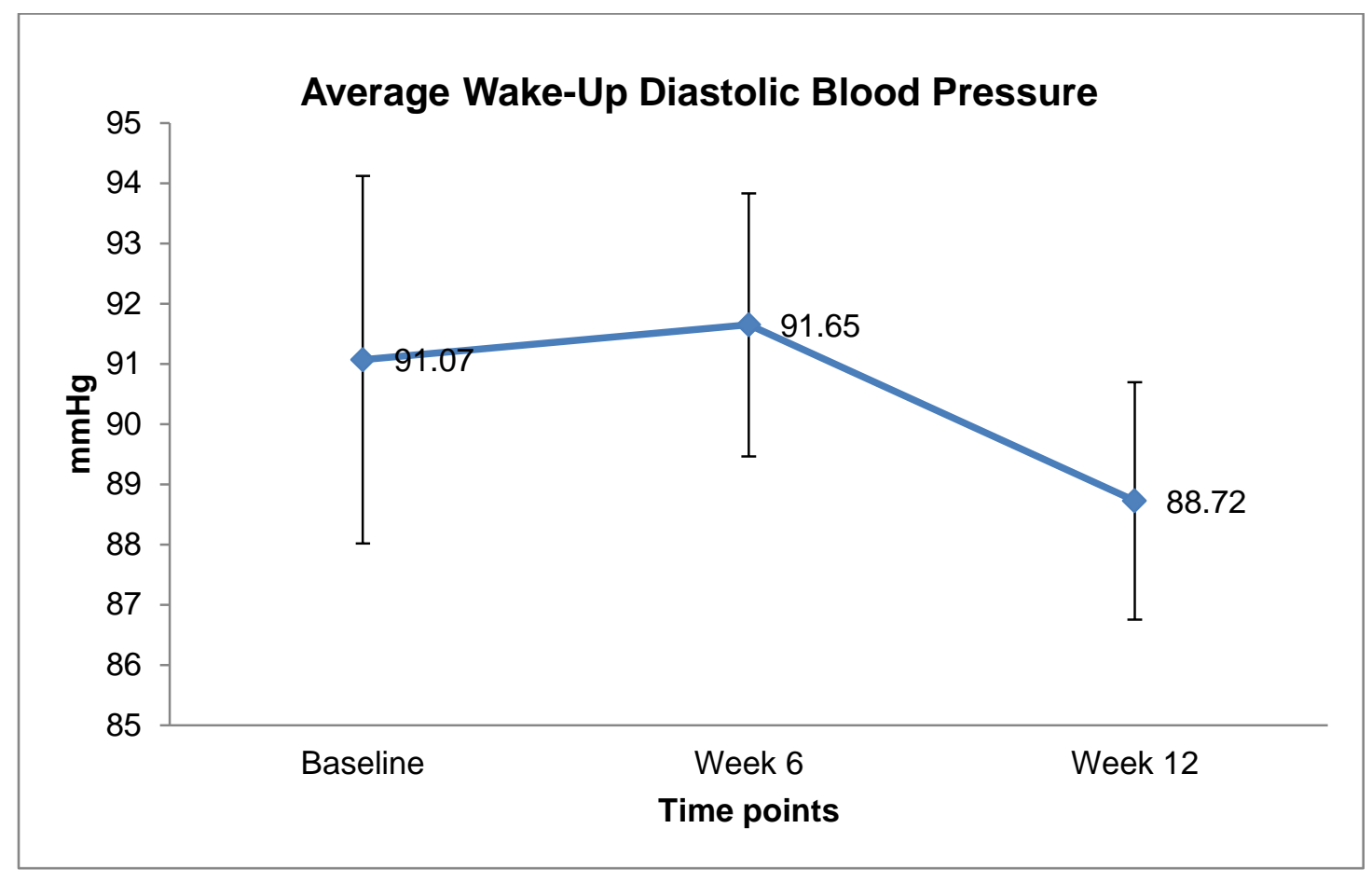

Figure 5. Comparison between baseline and Weeks 6 and 12 in Home Blood Pressure Measurements of Wake-Up Diastolic Blood Pressure

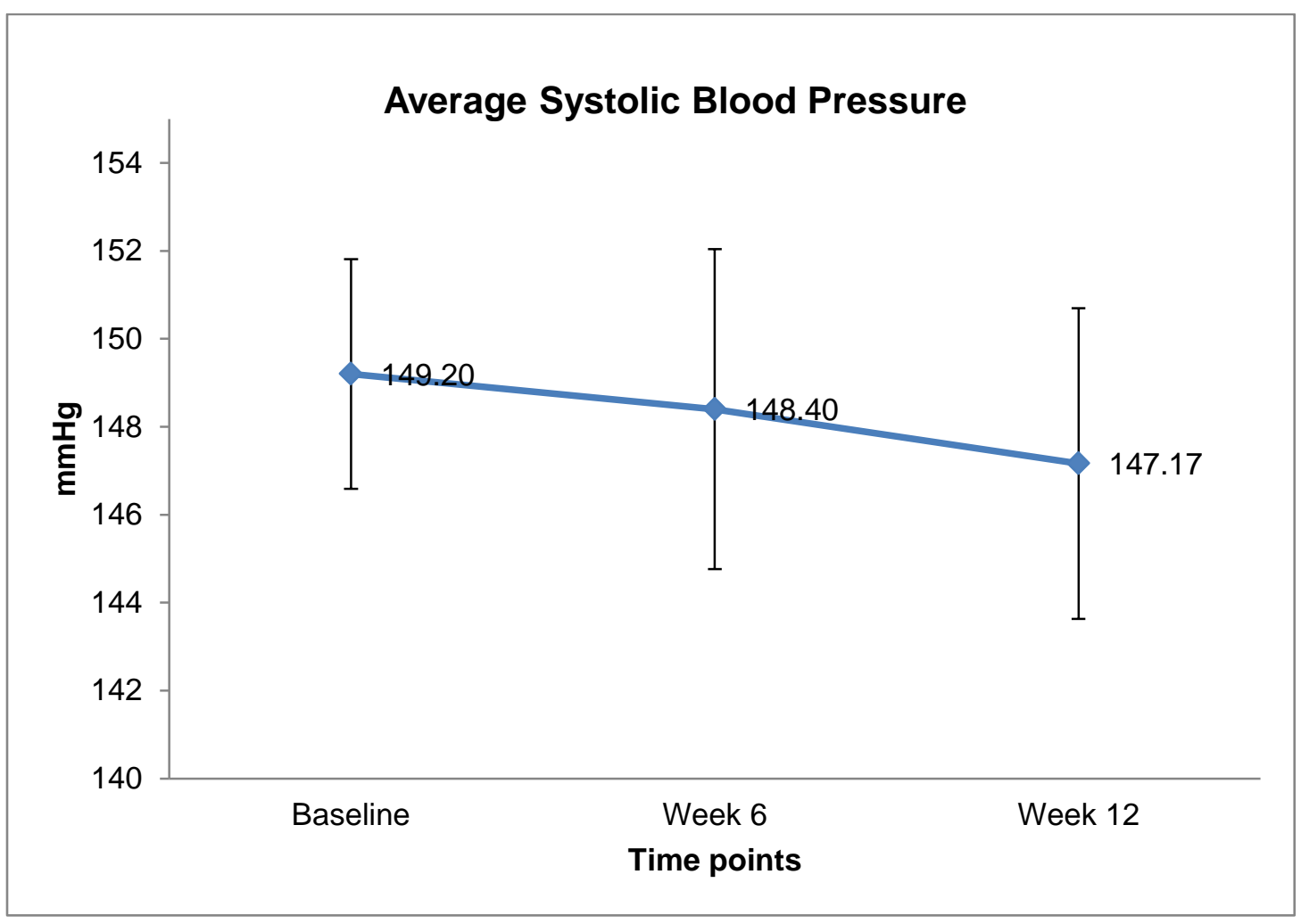

Figure 6. Comparison between baseline and Weeks 6 and 12 in Office Blood Pressure Measurements of Systolic Blood Pressure 
Table 5: Comparison between baseline and Week 12 in Office Blood Pressure Measurements (OBPM)

\begin{tabular}{|c|c|c|c|c|}
\hline $\begin{array}{l}\text { OFFICE BLOOD PRESSURE } \\
\text { MEASUREMENT (OBPM) }\end{array}$ & Mean & $\begin{array}{r}\text { Std. } \\
\text { Deviation }\end{array}$ & Sig. & $\begin{array}{l}\text { Percent } \\
\text { Change } \\
\text { (versus } \\
\text { Baseline) }\end{array}$ \\
\hline \multirow{2}{*}{ Systolic OBPM } & 149.20 & 8.254 & \multirow{2}{*}{0.520} & \multirow{2}{*}{$-1.36 \%$} \\
\hline & 147.17 & 11.170 & & \\
\hline \multirow{2}{*}{ Diastolic OBPM } & 92.23 & 5.946 & \multirow{2}{*}{0.304} & \multirow{2}{*}{$1.95 \%$} \\
\hline & 94.03 & 6.950 & & \\
\hline
\end{tabular}

The secondary objective was to evaluate the effect of the study product on glucose metabolism and lipid levels. Results demonstrated no significant changes in Fasting Glucose, Fasting Insulin, HbA1c, Total Cholesterol, HDL Cholesterol, LDL Cholesterol, 8-Isoprostane and Triglycerides. However, small non-significant reductions from baseline to Week 12 were observed in Fasting Glucose, Fasting Insulin, Total and LDL Cholesterol (Table 6). 8Isoprostane was increased by $20.36 \%$, although this change was non-significant.

Table 6: Comparison between baseline and Week 12 in Laboratory Assessments

\begin{tabular}{|c|c|c|c|}
\hline Laboratory Assessments & Mean & $\begin{array}{c}\text { Std. } \\
\text { Deviation }\end{array}$ & $\begin{array}{r}\text { Percent } \\
\text { Change (versus } \\
\text { Baseline) }\end{array}$ \\
\hline \multirow{2}{*}{ Fasting Glucose } & 94.9 & 15.096 & \multirow{2}{*}{$-0.53 \%$} \\
\hline & 94.4 & 15.565 & \\
\hline \multirow{2}{*}{ Fasting Insulin } & 16.06 & 14.107 & \multirow{2}{*}{$-5.29 \%$} \\
\hline & 15.21 & 6.954 & \\
\hline \multirow{2}{*}{$\mathrm{HbA} 1 \mathrm{c}$} & 5.57 & 0.291 & \multirow{2}{*}{$0.36 \%$} \\
\hline & 5.59 & 0.202 & \\
\hline \multirow{2}{*}{ Total Cholesterol } & 201.6 & 43.894 & \multirow{2}{*}{$-0.69 \%$} \\
\hline & 200.2 & 29.062 & \\
\hline \multirow{2}{*}{ LDL Cholesterol } & 118.1 & 34.398 & \multirow{2}{*}{$-1.86 \%$} \\
\hline & 115.9 & 25.632 & \\
\hline \multirow{2}{*}{ HDL Cholesterol } & 48.4 & 9.524 & \multirow{2}{*}{$-3.10 \%$} \\
\hline & 46.9 & 5.301 & \\
\hline \multirow{2}{*}{ Triglycerides } & 175.4 & 78.794 & \multirow{2}{*}{$6.67 \%$} \\
\hline & 187.1 & 82.941 & \\
\hline \multirow{2}{*}{ 8-Isoprostane } & 71.82 & 30.679 & \multirow{2}{*}{$20.36 \%$} \\
\hline & 86.44 & 29.308 & \\
\hline
\end{tabular}


No clinically significant changes were observed for the safety parameters, including vital signs, CBC, and CMP. Moreover, there were no serious adverse events reported in this study.

\section{DISCUSSION}

This study investigated the effects of a proprietary onion-pumpkin extract (Optain120) on blood pressure, blood glucose and lipid levels, which are all measurements for cardiovascular disease risk. The study was performed on healthy subjects with slightly elevated blood pressure and who fall into the clinical category of Stage 1 hypertension (Systolic BP 140-159, Diastolic BP 90-99). Patients in this risk category would most likely be recommended to lifestyle changes over pharmaceutical intervention, and incorporation of dietary supplements may be a beneficial addition to other dietary changes.

Blood pressure elicits diurnal variation, with a decrease during sleep and a surge upon waking [11,12]. While it is normal for blood pressure to suddenly spike in the morning, hypertensive individuals have a greater risk of sudden death, heart attack and stroke in the first 4 to 6 hours post-awakening [11,13]. Therefore, it is not surprising that a small study found patients with higher blood pressure in the morning have higher cardiovascular morbidity and mortality rates [14]. While only non-significant decreases were observed in blood pressure upon waking in this study, we found that supplementation of Optain120 for 12 weeks resulted in a significant reduction of systolic and diastolic blood pressure before bedtime.

Although the effects of morning blood pressure are better understood, it has been discovered that antihypertension medications taken at bedtime can improve ambulatory blood pressure control. In a prospective, open-label trial on patients with resistant hypertension, there was a significant reduction in sleep-time systolic and diastolic blood pressure, and an increased prevalence of controlled ambulatory blood pressure compared to all medications upon wakening [15], in addition to decreased cardiovascular morbidity and mortality rates [16,17]. Therefore, large scale trials targeting the evening blood pressure surge are necessary, as the evening surge may affect cardiovascular disease-related morbidity and mortality.

We screened various vegetables, including pumpkin, onion, asparagus, eggplant, celery and tomato, for ACE inhibitory activity. Although asparagus exhibited the highest inhibiting effect, we discovered that the combination of pumpkin and onion extracts exhibited the highest synergistic effect on ACE inhibition (unpublished results, Table 7). Furthermore, the synergistic effect was confirmed using spontaneously hypertensive rats [18]. Pumpkin is expected to have a variety of health benefits based on its nutrient profile, and preliminary studies in animal models. Pumpkin is rich in carotenoids, including lutein, carotene, and beta-carotene [19]. It also contains acyl-coenzyme oxidase (ACOX), an enzyme that catalyzes fatty acid oxidation, specifically the oxidation of fatty acid CoA esters [20]. Phenolic antioxidants found in pumpkins have also been reported to have applications in dietary strategies against hyperglycemia and related complications, including cellular oxidative stress and hypertension [10]. A number of animal studies demonstrated that pumpkin extracts, as well as polysaccharides from pumpkin fruit, can reduce hyperglycemia in normal and diabetic rats [21-26]. The compounds trigonelline and nicotinic acid are found in pumpkin paste, and are thought to improve glucose tolerance. When either purified component (trigonelline or nicotinic acid) or whole pumpkin paste concentrate were fed to non-obese diabetic rats, improved glucose tolerance and gradual increase in insulin 
was observed [22]. Also, among the constituents identified in pumpkin, nicotianamine showed a potent ACE inhibitory effect in published literature [27]. Thus we hypothesized that nicotianamine might be contributing the blood pressure lowering effect in OPtain 120.

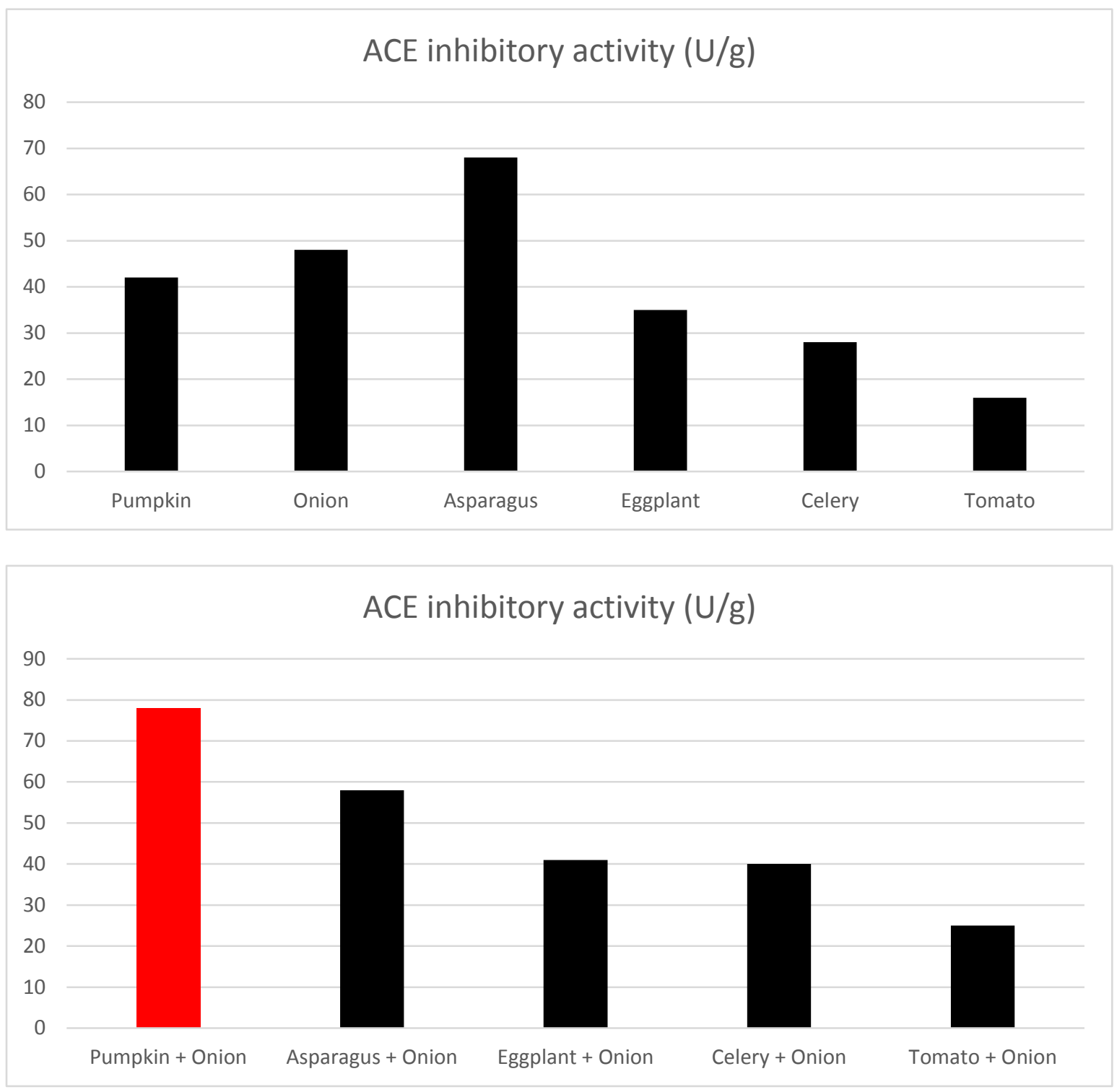

Figure 7. Potencies in ACE inhibitory activity of various vegetables such as pumpkin, onion, asparagus, eggplant, celery and tomato are shown (upper figure). In the combination experiment (lower figure), it is clear that the pumpkin and onion extracts mixture showed the highest ACE inhibitory effect.

Despite an attractive nutrient profile and encouraging animal studies, extensive clinical trials on pumpkin are lacking. One human trial demonstrated that oral administration of extract from the related squash Cucurbita ficifolia significantly decreased blood glucose levels of diabetic patients [28]. This benefit is attributed to active compounds which may be shared with pumpkin: flavonoids, alkaloids, and polyphenolic compounds, as well as glutathione peroxidase and superoxide dismutase [29]. While animal studies show that pumpkin flesh extracts improve the glycemic status of diabetic animals, studies evaluating the effect of pumpkin flesh on blood 
pressure have not been performed. Another pumpkin product, pumpkin seed oil, has been effectively used in blood pressure studies. Pumpkin seed oil supplementation lowers blood pressure and improves lipid profile in animal studies [8]. In fact, hypertensive rats supplemented with pumpkin seed oil received health benefits, with their blood pressure and NO metabolites, comparable to the antihypertensive drug amlopidine (a calcium channel blocker) [30]. In another animal model of hypertensive rats, pumpkin seed oil, followed by administration of the calcium antagonist felodipine or the ACE inhibitor captopril, produced significant hypotensive effects [31]. Similar to these results, a recent clinical trial involving postmenopausal women found that 12-week administration of $2 \mathrm{~g}$ /day pumpkin seed oil led to a significant increase in HDL cholesterol and a significant decrease in diastolic blood pressure [9].

Another food showing great promise for reducing hypertension is onion. Onion contains essential oils, flavonoids or polyphenolic compounds with antioxidant properties [32], and sulfur compounds such as cysteine sulfoxide known to exhibit antimicrobial effects [33]. However, the most potent hypertension-combating compound found in onions is quercetin. Onion contains high levels of quercetin, a flavonol, [34-36] as high as $0,25-0.5 \mathrm{~g} / \mathrm{kg}$ [37], which can significantly decrease blood triglyceride, and glucose concentrations [38]. In a randomized, controlled design, supplementation of quercetin has been demonstrated to reduce blood pressure in hypertensive rodents [39] and men and women with prehypertension and stage 1 hypertension [40], in addition to overweight or obese subjects [41]. It has been proposed that quercetin reduces blood pressure through several mechanisms, including antioxidant effects, inhibition of angiotensin-converting enzyme activity, and improved endothelium-dependent and -independent function [35].

Onions have been the subject of several clinical trials, all of which suggest that an onion can help maintain healthy blood pressure and metabolism in vivo. For example, systolic and diastolic blood pressures were significantly decreased in healthy male smokers who consumed quercetinrich onion peel extract, compared to those who consumed placebo [42]. Furthermore, these patients also experienced significant decreases in total cholesterol, LDL cholesterol and glucose concentrations after 10 weeks of supplementation. Another randomized, placebo-controlled, double-blind trial demonstrated a significant decrease in arterial blood pressure and reduction in plasma viscosity 5 hours after being administered with a formulation containing onions and olive oil [6]. Furthermore, preclinical and clinical studies have shown that supplementation with onion peel extract benefits both lipid profile and glucose levels [43, 42].

In contrast to the above animal and human studies, which investigated the effects of pumpkin and onion separately, the current study did not produce significant changes in glucose, insulin, HbA1c, or 8-isoprostane. This result is not unexpected. Unlike previous human and animal subjects, the subjects of the current study were not diabetic and did not exhibit elevate glucose levels at baseline. However, toward the end of the 12-week study period, there were small, non-significant reductions from baseline in glucose and insulin levels, as well as in total cholesterol and LDL cholesterol. Although these changes were not statistically significant, 8isoprostane levels were elevated by approximately 20\%. 8-isoprostane serves as a marker of oxidant stress and acts as a vasoconstrictor in vivo. However, the increased levels of 8isoprostane measured in this study do not appear to significantly affect vascular function; several other measurements of cardiovascular health were also found to react positively to OPtain120. 
Nonetheless, the current study confirms an important result: OPtain120 produced a nearlysignificant diastolic and significant systolic blood pressure reduction before bedtime. Additionally, decreases in systolic and diastolic HBPM and in systolic OBPM were observed, although they were not statistically significant. Moreover, this is the first study to present the combined effects of pumpkin and onion towards reducing blood pressure.

The reduction of hypertension has a significant impact on patient outcomes, since current treatment options are far from ideal. Interventions for hypertensive patients rely primarily on lifestyle changes, which requires a high level of patient commitment, or on antihypertensive medications, which have significant side effects.

This study provides preliminary evidence that the proprietary onion-pumpkin extract (OPtain120) may promote healthy blood pressure. Randomized, placebo-controlled clinical studies in larger populations are required to further determine the effects of this extract (OPtain120).

\section{CONCLUSION}

The proprietary onion-pumpkin extract (OPtain120) was safely consumed for a 12-week period. OPtain120 appears to be effective in lowering systolic blood pressure at bedtime in healthy individuals with slightly elevated blood pressure and who also manage their cardiovascular risk factors with diet and lifestyle.

List of abbreviations used: $\mathrm{ACE}$, angiotensin converting enzyme; $\mathrm{ARB}$, angiotensin receptor blocker; BB, $\beta$-blocker; CCB, calcium channel blockers; ALLHAT, Antihypertensive and Lipidlowering Treatment to prevent Heart Attack; NO, nitric oxide; HBPM, Home Blood Pressure Measurements; OBPM, Office Blood Pressure Measurement; LDL, low-density lipoprotein; HDL, high -density lipoprotein; CRO, contract research organization; GMP, good manufacturing practices; ACOX, acyl-coenzyme oxidase.

\section{Authors' contributions}

JU was the Principal Investigator and together with OY, HM, YS and XC, contributed to the writing, data analyses, and data interpretation that are part of this manuscript.

Funding: The present study was supported by Ryusendo Co., Ltd., Tokyo 171-0021

\section{REFERENCES}

1. Obineche EN: Management of hypertension: update and review. Bulletin of The Kuwait Institute For Medical Specialization 2003; 2:73-82.

2. Major outcomes in high-risk hypertensive patients randomized to angiotensin-converting enzyme inhibitor or calcium channel blocker vs diuretic: The Antihypertensive and LipidLowering Treatment to Prevent Heart Attack Trial (ALLHAT). JAMA 2002; 288:29812997.

3. Houston MC: Nutrition and nutraceutical supplements in the treatment of hypertension. Expert Rev Cardiovasc Ther 2010; 8:821-833. 
4. Yamamoto Y, Aoyama S, Hamaguchi N, Rhi GS: Antioxidative and antihypertensive effects of Welsh onion on rats fed with a high-fat high-sucrose diet. Biosci Biotechnol Biochem 2005; 69:1311-1317.

5. Sakai Y, Murakami T, Yamamoto Y: Antihypertensive effects of onion on NO synthase inhibitor-induced hypertensive rats and spontaneously hypertensive rats. Biosci Biotechnol Biochem 2003; 67:1305-1311.

6. Kalus U, Pindur G, Jung F, Mayer B, Radtke H, Bachmann K, Mrowietz C, Koscielny J, Kiesewetter $\mathrm{H}$ : Influence of the onion as an essential ingredient of the Mediterranean diet on arterial blood pressure and blood fluidity. Arzneimittelforschung 2000; 50:795-801.

7. Jafarian A, Zolfaghari B, Parnianifard M: The effects of methanolic, chloroform, and ethylacetate extracts of the Cucurbita pepo L. on the delay type hypersensitivity and antibody production. Res Pharm Sci 2012; 7:217-224.

8. Gossell-Williams M, Lyttle K, Clarke T, Gardner M, Simon O: Supplementation with pumpkin seed oil improves plasma lipid profile and cardiovascular outcomes of female non-ovariectomized and ovariectomized Sprague-Dawley rats. Phytother Res 2008; 22:873-877.

9. Gossell-Williams M, Hyde C, Hunter T, Simms-Stewart D, Fletcher H, McGrowder D, Walters CA: Improvement in HDL cholesterol in postmenopausal women supplemented with pumpkin seed oil: pilot study. Climacteric 2011; 14:558-564.

10. Kwon YI, Apostolidis E, Kim YC, Shetty K: Health benefits of traditional corn, beans, and pumpkin: in vitro studies for hyperglycemia and hypertension management. $J$ Med Food 2007; 10:266-275.

11. Muller JE, Tofler GH, Stone PH: Circadian variation and triggers of onset of acute cardiovascular disease. Circulation 1989; 79:733-743.

12. Pickering TG, Shimbo D, Haas D: Ambulatory blood-pressure monitoring. $N$ Engl J Med 2006; 354:2368-2374.

13. Kario K, Pickering TG, Umeda Y, Hoshide S, Hoshide Y, Morinari M, Murata M, Kuroda T, Schwartz JE, Shimada K: Morning surge in blood pressure as a predictor of silent and clinical cerebrovascular disease in elderly hypertensives a prospective study. Circulation 2003; 107:1401-1406.

14. Gosse P, Lasserre R, Minifie C, Lemetayer P, Clementy J: Blood pressure surge on rising. J Hypertens 2004; 22:1113-1118.

15. Ayala DE, Hermida RC, Mojon A, Fernandez JR: Cardiovascular risk of resistant hypertension: dependence on treatment-time regimen of blood pressure-lowering medications. Chronobiol Int 2013; 30:340-352.

16. Hermida RC, Ayala DE, Mojon A, Fernandez JR: Influence of time of day of blood pressure-lowering treatment on cardiovascular risk in hypertensive patients with type 2 diabetes. Diabetes Care 2011; 34:1270-1276.

17. Hermida RC, Ayala DE, Mojon A, Fernandez JR: Influence of circadian time of hypertension treatment on cardiovascular risk: results of the MAPEC study. Chronobiol Int 2010: 27:1629-1651.

18. Saito Y, Yoshinari O, Igarashi K. Antihypertensive Effect of combined administration of onion extract and pumpkin extract in spontaneously hypertensive rats and hypertensive patients. Rinsho Iyaku, 2004; 20:593-603.

19. Veronezi CM, Jorge N: Bioactive compounds in lipid fractions of pumpkin (Cucurbita sp) seeds for use in food. J Food Sci 2012; 77:C653-657.

20. De Bellis L, Gonzali S, Alpi A, Hayashi H, Hayashi M, Nishimura M: Purification and characterization of a novel pumpkin short-chain acyl-coenzyme A oxidase with structural similarity to acyl-coenzyme A dehydrogenases. Plant Physiol 2000; 123:327-334. 
21. Diaz-Flores M, Angeles-Mejia S, Baiza-Gutman LA, Medina-Navarro R, HernandezSaavedra D, Ortega-Camarillo C, Roman-Ramos R, Cruz M, Alarcon-Aguilar FJ: Effect of an aqueous extract of Cucurbita ficifolia Bouche on the glutathione redox cycle in mice with STZ-induced diabetes. J Ethnopharmacol 2012; 144:101-108.

22. Yoshinari O, Sato H, Igarashi K: Anti-diabetic effects of pumpkin and its components, trigonelline and nicotinic acid, on Goto-Kakizaki rats. Biosci Biotechnol Biochem 2009; 73:1033-1041.

23. Xia T, Wang Q: Antihyperglycemic effect of Cucurbita ficifolia fruit extract in streptozotocin-induced diabetic rats. Fitoterapia 2006; 77:530-533.

24. Alarcon-Aguilar FJ, Hernandez-Galicia E, Campos-Sepulveda AE, Xolalpa-Molina S, Rivas-Vilchis JF, Vazquez-Carrillo LI, Roman-Ramos R: Evaluation of the hypoglycemic effect of Cucurbita ficifolia Bouche (Cucurbitaceae) in different experimental models. $J$ Ethnopharmacol 2002; 82:185-189.

25. Quanhong L, Caili F, Yukui R, Guanghui H, Tongyi C: Effects of protein-bound polysaccharide isolated from pumpkin on insulin in diabetic rats. Plant Foods Hum Nutr 2005, 60:13-16.

26. Baldi A, Chaudhary N, Maru J, Joshi R: Effect of pumpkin concentrate on alloxan induced diabetic rats. Journal of Global Pharma Technology 2010; 2:24-27.

27. Itagaki S, Uchiyama M, Kanazawa S, Hosoi K, Terui K, Shimoyama R, Tsuchida S, Ueno $\mathrm{S}$, Hayakari M: Identification of the angiotensin converting enzyme inhibitors in the local product of Aomori prefecture. Hirosaki Med J 2013; 64:50-57.

28. Acosta-Patino JL, Jimenez-Balderas E, Juarez-Oropeza MA, Diaz-Zagoya JC: Hypoglycemic action of Cucurbita ficifolia on Type 2 diabetic patients with moderately high blood glucose levels. J Ethnopharmacol 2001; 77:99-101.

29. Bayat A, Jamali Z, Hajianfar H, Heidari Beni M: Effects of <i>Cucurbita ficifolia</i> Intake on Type 2 Diabetes: Review of Current Evidences. Shiraz E-Med J 2014; 15:e20586.

30. El-Mosallamy AE, Sleem AA, Abdel-Salam OM, Shaffie N, Kenawy SA: Antihypertensive and cardioprotective effects of pumpkin seed oil. J Med Food 2012; 15:180-189.

31. Zuhair HA, Abd El-Fattah AA, El-Sayed MI: Pumpkin-seed oil modulates the effect of felodipine and captopril in spontaneously hypertensive rats. Pharmacol Res 2000; 41:555563.

32. Perez-Gregorio MR, Regueiro J, Simal-Gandara J, Rodrigues AS, Almeida DP: Increasing the added-value of onions as a source of antioxidant flavonoids: a critical review. Crit Rev Food Sci Nutr 2014; 54:1050-1062.

33. Sagdic O, Tornuk F: Antimicrobial Properties of Organosulfur Compounds. In Dietary Phytochemicals and Microbes. Edited by Patra AK: Springer Netherlands; 2012: 127-156.

34. Galindo P, Rodriguez-Gómez I, González-Manzano S, Dueñas M, Jiménez R, Menéndez C, Vargas F, Tamargo J, Santos-Buelga C, Pérez-Vizcaíno F: Glucuronidated quercetin lowers blood pressure in spontaneously hypertensive rats via deconjugation. PloS one 2012; 7:e32673.

35. Larson AJ, Symons JD, Jalili T: Therapeutic potential of quercetin to decrease blood pressure: review of efficacy and mechanisms. Adv Nutr 2012; 3:39-46.

36. Toh JY, Tan VH, Lim PY, Lim ST, Chong MF: Flavonoids from Fruit and Vegetables: A Focus on Cardiovascular Risk Factors. Current Atherosclerosis Reports 2013; 15:1-7.

37. Hertog MG, Feskens EJ, Hollman PC, Katan MB, Kromhout D: Dietary antioxidant flavonoids and risk of coronary heart disease: the Zutphen Elderly Study. Lancet 1993; 342:1007-1011. 
38. Lee SM, Moon J, Chung JH, Cha YJ, Shin MJ: Effect of quercetin-rich onion peel extracts on arterial thrombosis in rats. Food Chem Toxicol 2013; 57:99-105.

39. Duarte J, Perez-Palencia R, Vargas F, Ocete MA, Perez-Vizcaino F, Zarzuelo A, Tamargo $\mathrm{J}$ : Antihypertensive effects of the flavonoid quercetin in spontaneously hypertensive rats. Br J Pharmacol 2001; 133:117-124.

40. Edwards RL, Lyon T, Litwin SE, Rabovsky A, Symons JD, Jalili T: Quercetin reduces blood pressure in hypertensive subjects. J Nutr 2007; 137:2405-2411.

41. Egert S, Bosy-Westphal A, Seiberl J, Kurbitz C, Settler U, Plachta-Danielzik S, Wagner AE, Frank J, Schrezenmeir J, Rimbach G, et al: Quercetin reduces systolic blood pressure and plasma oxidised low-density lipoprotein concentrations in overweight subjects with a high-cardiovascular disease risk phenotype: a double-blinded, placebo-controlled crossover study. Br J Nutr 2009; 102:1065-1074.

42. Lee KH, Park E, Lee HJ, Kim MO, Cha YJ, Kim JM, Lee H, Shin MJ: Effects of daily quercetin-rich supplementation on cardiometabolic risks in male smokers. Nutr Res Pract 2011; 5:28-33.

43. Matsunaga S, Azuma K, Watanabe M, Tsuka T, Imagawa T, Osaki T, Okamoto Y: Onion peel tea ameliorates obesity and affects blood parameters in a mouse model of high-fatdiet-induced obesity. Exp Ther Med 2014; 7:379-382.

44. Kim J, Cha YJ, Lee KH, Park E: Effect of onion peel extract supplementation on the lipid profile and antioxidative status of healthy young women: a randomized, placebocontrolled, double-blind, crossover trial. Nutr Res Pract 2013; 7:373-379. 